\title{
Carbohydrate drugs: current status and development prospect
}

\author{
Yan Zhang ${ }^{1}$, Fengshan Wang ${ }^{1,2, *}$ \\ ${ }^{1}$ Key Laboratory of Chemical Biology of Natural Products (Ministry of Education), Institute of Biochemical and Biotechnological \\ Drug, School of Pharmaceutical Sciences, Shandong University, Ji'nan, Shandong, China; \\ ${ }^{2}$ National Glycoengineering Research Center, Shandong University, Ji'nan, Shandong, China.
}

\begin{abstract}
Summary In recent years, there has been a great effort devoted to the investigation of the roles of carbohydrates in various essential biological processes and the development of carbohydrates to therapeutic drugs. This review summarizes the carbohydrate drugs which have been recorded in several pharmacopoeias, marketed, and under development. A prospect of the future development of carbohydrate drugs is discussed as well.
\end{abstract}

Keywords: Carbohydrate drugs, pharmacopoeia, carbohydrate modification, carbohydrate vaccine, oligosaccharide synthesis

\section{Introduction}

Carbohydrates are the essential constituents of organisms and play crucial roles in many important physiological and pathogenic processes, such as cell surface recognition, signal transduction, tumor metastasis, etc. However, carbohydrates, the third major class of biopolymers, have drawn much less attention from a drug development perspective compared to nucleic acids and proteins due to our very recent understanding of the fundamental glycobiology $(1,2)$. Nevertheless, carbohydrate drugs still pave the way to a non-ignorable area of the world of therapeutics owing to their natural significance and the vast effort devoted by glycoscientists in recent decades.

This review mainly focuses on carbohydrate drugs, including polysaccharides, oligosaccharides, monosaccharides and their derivatives. The status of carbohydrate drugs included in several pharmacopoeias, marketed, or under development is summarized and the development prospect of carbohydrate drugs is discussed.

\section{Carbohydrate drugs included in important pharmacopoeias}

To compile the recorded carbohydrate drugs, four pharmacopoeias including United States Pharmacopoeia

\footnotetext{
*Address correspondence to:

Dr. Fengshan Wang, Institute of Biochemical and Biotechnological Drug, School of Pharmaceutical Sciences, Shandong University, Ji'nan 250012, Shandong, China.

E-mail: fswang@sdu.edu.cn
}

37/National Formulary 32 (USP 37/NF 32), European Pharmacopoeia 7.0 (EP 7.0), Japanese Pharmacopoeia Sixteenth Edition (JP 16), Chinese Pharmacopoeia 2010 (CP 2010) and Chinese ministerial standards 1998 were meticulously searched and the acquired data was analyzed (3-7). A total of 131 carbohydrate drugs have been included in the four pharmacopoeias, demonstrating the fundamental role of carbohydrate pharmaceuticals both in crude drugs and pharmaceutical excipients $(8,9)$. As indicated in Figure 1A, polysaccharides derived from natural animal, plant and microorganism sources still

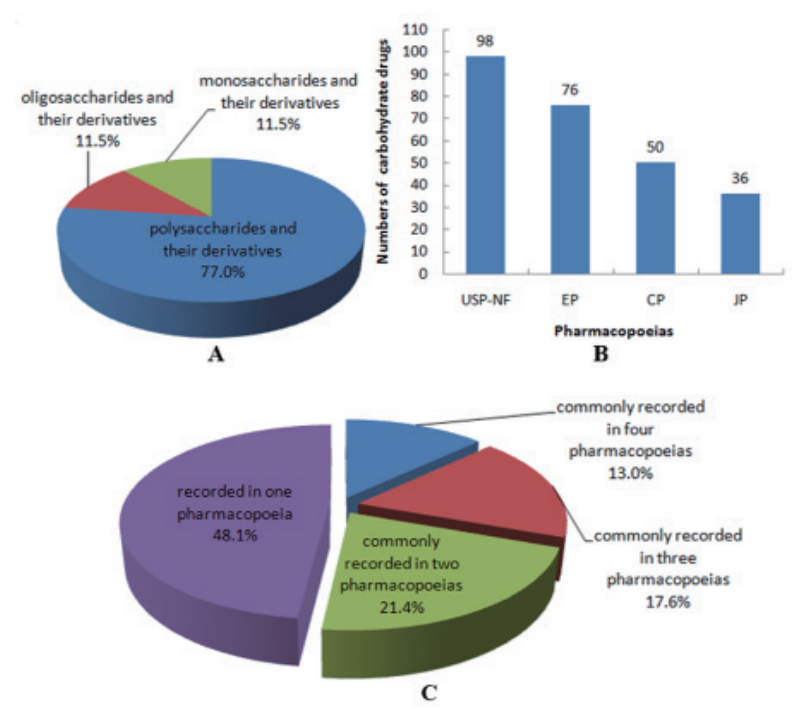

Figure 1. Data analysis of carbohydrate drugs recorded in USP, EP, JP, and CP. (A) Composition of carbohydrate drugs recorded in the pharmacopoeias. (B) Numbers of carbohydrate drugs recorded in each pharmacopoeia. (C) Carbohydrate drugs commonly recorded in the pharmacopoeias. 
Table 1. Carbohydrate drugs commonly recorded in four or three of the pharmacopoeias

\begin{tabular}{|c|c|c|c|c|c|}
\hline \multirow{2}{*}{ Drugs } & \multirow{2}{*}{ Pharmacopoeias } & \multicolumn{3}{|c|}{ Dosage forms } & \multirow{2}{*}{ Structures } \\
\hline & & USP & $\mathrm{CP}$ & JP & \\
\hline Acacia & USP EP CP JP & $\mathrm{PE}$ & $\mathrm{PE}$ & $\mathrm{CD}$ & $\mathrm{P}$ \\
\hline Dextran 40 & USP EP CP JP & BD I & BD I & I & $\mathrm{P}$ \\
\hline Dextran 70 & USP EP CP JP & BD I & BD I & $\mathrm{I}$ & $\mathrm{P}$ \\
\hline Dextrin & USP EP CP JP & PE & $\mathrm{PE}$ & & $\mathrm{P}$ \\
\hline Cellacefate & USP EP CP JP & $\mathrm{PE}$ & PE & & $\mathrm{P}$ \\
\hline Corn Starch & USP EP CP JP & $\mathrm{PE}$ & PE & & $\mathrm{P}$ \\
\hline Fructose & USP EP CP JP & PE BD I & $\mathrm{PE}$ & $\mathrm{I}$ & M \\
\hline Heparin Sodium & USP EP CP JP & BD I S & BD I CR & & $\mathrm{P}$ \\
\hline Hydroxypropyl Cellulose & USP EP CP JP & $\mathrm{PE}$ & $\mathrm{PE}$ & & $\mathrm{P}$ \\
\hline Hypromellose & USP EP CP JP & PE BD OPS & PE & & $\mathrm{P}$ \\
\hline Lactose Monohydrate & USP EP CP JP & $\mathrm{PE}$ & PE & & $\mathrm{O}$ \\
\hline Lactulose & USP EP CP JP & BD S & OS & & $\mathrm{O}$ \\
\hline Meglumine & USP EP CP JP & PE BD & $\mathrm{BD}$ & & M \\
\hline Methylcellulose & USP EP CP JP & PE BD OPS OS T & $\mathrm{PE}$ & & $\mathrm{P}$ \\
\hline Microcrystalline Cellulose & USP EP CP JP & $\mathrm{PE}$ & $\mathrm{PE}$ & & $\mathrm{P}$ \\
\hline Sucralfate & USP EP CP JP & $\mathrm{BD} \mathrm{T}$ & BD OSU DT CT C & & $\mathrm{O}$ \\
\hline Sucrose & USP EP CP JP & $\mathrm{PE}$ & $\mathrm{PE}$ & & $\mathrm{O}$ \\
\hline Alginic Acid & USP EP CP & PE & $\mathrm{PE}^{*}$ & & $\mathrm{P}$ \\
\hline Betadex & USP EP CP & $\mathrm{PE}$ & $\mathrm{PE}$ & & $\mathrm{P}$ \\
\hline Carboxymethylcellulose Sodium & USP EP CP & PE BD T P & PE & & $\mathrm{P}$ \\
\hline Cellulose Acetate & USP EP CP & $\mathrm{PE}$ & PE & & $\mathrm{P}$ \\
\hline Croscarmellose Sodium & USP EP CP & $\mathrm{PE}$ & $\mathrm{PE}$ & & $\mathrm{P}$ \\
\hline Ethylcellulose & USP EP CP & $\mathrm{PE}$ & $\mathrm{PE}$ & & $\mathrm{P}$ \\
\hline Glucose Monohydrate & USP EP CP & PE BD I & BD I & & M \\
\hline Heparin Calcium & USP EP CP & BD I & $\mathrm{BD}^{*} \mathrm{I}^{*}$ & & $\mathrm{P}$ \\
\hline Hydroxypropyl Betadex & USP EP CP & $\mathrm{PE}$ & $\mathrm{PE}$ & & $\mathrm{P}$ \\
\hline Maltodextrin & USP EP CP & PE & PE & & $\mathrm{P}$ \\
\hline Pregelatinized Starch & USP EP CP & PE & PE & & $\mathrm{P}$ \\
\hline Sodium Alginate & USP EP CP & $\mathrm{PE}$ & PE & & $\mathrm{P}$ \\
\hline Xanthan Gum & USP EP CP & PE & PE & & $\mathrm{P}$ \\
\hline Agar & USPCP JP & PE & PE & CD & $\mathrm{P}$ \\
\hline Simple Syrup & USPCP JP & PE & $\mathrm{PE}$ & & $\mathrm{O}$ \\
\hline Anhydrous Glucose & EP CP JP & & BD I & I & M \\
\hline Anhydrous Lactose & USP EP JP & $\mathrm{PE}$ & & & $\mathrm{O}$ \\
\hline Hypromellose Phthalate & USP EP JP & PE & & & $\mathrm{P}$ \\
\hline Potato Starch & USP EP JP & PE & & & $\mathrm{P}$ \\
\hline Powderd Cellulose & USP EP JP & $\mathrm{PE}$ & & & $\mathrm{P}$ \\
\hline Rice Starch & USP EP JP & PE & & & $\mathrm{P}$ \\
\hline Tragacanth & USP EP JP & PE & & & $\mathrm{P}$ \\
\hline Wheat Starch & USP EP JP & PE & & & $\mathrm{P}$ \\
\hline
\end{tabular}

*, collected by Chinese ministerial standards 1998; USP, United States Pharmacopoeia; EP, European Pharmacopoeia; CP, Chinese Pharmacopoeia; JP, Japanese Pharmacopoeia; PE, pharmaceutical excipient; BD, bulk drug; T, tablet; C, capsule; DT, dispersible tablet; CT, chewable tablet; CR, cream; I, injection; S, solution; OS, oral solution; OSU, oral suspension; OPS, ophthalmic solution; CD, crude drug; M, monosaccharide or its derivative; O, oligosaccharide or its derivative; P, polysaccharide or its derivative. Dosage forms are not recorded in EP, and $\mathrm{PE}$ and $\mathrm{BD}$ are not distinguished in EP and JP.

represent the largest percentage of more than two thirds of all the recorded carbohydrate drugs $(101,77.0 \%)$, followed by oligosaccharides, monosaccharides and their derivatives, contributing an equal occupation of $11.5 \%$ (15).

The comparison of the numbers of carbohydrate drugs recorded by each pharmacopoeia reveals that USPNF covers the largest number of 98 , followed by 76 of EP, 50 of CP, and 36 of JP, respectively (Figure 1B). Among which, most carbohydrate drugs (63, 48.1\%) are recorded in only one of the four pharmacopoeias and only 17 (13.0\%) carbohydrate drugs are commonly recorded in all of the four pharmacopeias with $23(17.6 \%)$ carbohydrate drugs commonly recorded in three of them as shown in Figure 1C and Table 1.
In the course of analyzing the differences among carbohydrate drugs recorded by different pharmacopoeias, we noted that instead of entirely different active ingredients, diverse modifications of carbohydrate drugs such as salinization, esterification, and derivatization contribute most to the diversity of each pharmacopoeia $(10,11)$. For example, powdered cellulose is recorded in JP only while celluloseacetate is commonly recorded in all of the other three pharmacopoeias. Although hydroxypropyl cellulose is commonly recorded in all of the four pharmacopoeias, low-substituted hydroxypropyl cellulose is missed in EP compared with USP and JP. Even USP which records the most carbohydrate drugs contains glucose monohydrate only while anhydrous glucose is commonly collected by all the other three 
Table 2. Carbohydrate drugs marketed

\begin{tabular}{|c|c|c|}
\hline Drug (Trade name) & Country & Indications \\
\hline Astragalus polysaccharide & China & Cancer \\
\hline Coriolus versicolor polysaccharide & Japan & Cancer \\
\hline Ginseng polysaccharide & China & Cancer \\
\hline Lentinan & Japan & Cancer, hepatitis B \\
\hline Pachyman & China & Cancer \\
\hline Tremella polysaccharide & China & Cancer, chronic bronchitis \\
\hline Hydroxyethyl starch 130/0.4 (Voluven) & Germany & Blood volume expansion \\
\hline Hydroxyethyl starch 200/0.5 (Haes) & Germany & Blood volume expansion \\
\hline Fondaparinux sodium (Arixtra) & England & Thrombus \\
\hline Swine duodenum mucopolysaccharide & China & Coronary atherosclerosis \\
\hline Icodextrin (Extraneal) & Germany & Peritoneal dialysis \\
\hline Hai Kun Shen Xi Capsule & China & Chronic renal failure \\
\hline Pentosan polysulfate sodium (Elmiron) & America & Interstitial cystitis \\
\hline Mucopolysaccharide polysulfate (Hirudoid) & Germany & Phlebitis \\
\hline Zanamivir & England & Influenza \\
\hline Miglitol & Germany & Diabetes \\
\hline Testa triticum tricum purify (Fiberform) & Sweden & Constipation \\
\hline Polysacharidum of G. lucidum Karst & China & Neurosis, polymyositis \\
\hline
\end{tabular}

pharmacopoeias. In terms of molecular weight and the degree of polymerization, dextran 40 and dextran 70 are commonly recorded in all of the four pharmacopoeias while dextran 20 and dextranomer are recorded in CP and EP, respectively. Besides, broader sources and more sophisticated categories also make contribution to the diversity of the recorded carbohydrate drugs. Starches from corn, potato, rice, wheat, tapioca, and pea are all recorded in USP, while tapioca starch is not accepted by EP or JP, and only tapioca and corn starch are accepted by CP. Heparin sodium is also commonly recorded in all of the four pharmacopoeias, while in USP heparin sodium is obtained from the intestinal mucosa or other suitable tissues of domestic mammals used for food by human, and the EP heparin sodium is prepared either from the lungs of cattle or from the intestinal mucosa of pigs, cattle or sheep, and the CP heparin sodium comes from the intestinal mucosa of pigs or cattle, and the JP heparin is obtained from the livers, the lungs and the intestinal mucosa of healthy edible animals. For the different degrees of categories, sodium starch glycolate is categorized into type A, B and C in USP and EP according to their different ranges of $\mathrm{pH}$ and contents of sodium, while CP records just one type which has the same range of $\mathrm{pH}$ but different content of sodium from type A.

Additionally, in certain countries, some carbohydrate drugs commonly recorded in the foreign pharmacopoeias have already been used in clinic although have not been recorded in the native pharmacopoeia. On the other hand, certain commonly recorded carbohydrate drugs have not yet been used in clinic in other countries. Besides, some recorded pharmaceutical excipients with excellent properties have only been used as food additives in other countries.

Hence, in spite of the considerable number and diversity of carbohydrate drugs recorded in the pharmacopoeias, more comprehensive and normalized standards for carbohydrate drugs are urgently needed to accelerate their internationalized utilization. As far as we concerned, adoption and quotation of uncommonly recorded carbohydrate drugs especially for the diverse carbohydrate derivatives among all the pharmacopoeias are feasible ways to achieve this goal.

\section{Carbohydrate drugs marketed}

Except for the pharmacopoeia recorded carbohydrate drugs, numbers of carbohydrate drugs have already been marketed and used in clinic for years. We combed through the databases of Chinese Food and Drug Administration (CFDA) and United States Food and Drug Administration (FDA) for carbohydrate drugs marketed without included in the pharmacopoeias and eighteen of them were found (Table 2) (12-14). Their indications comprise a vast array of maladies ranging from cancer to constipation.

Thirty three percentage of the marketed carbohydrate drugs are exploited as clinical antitumor adjuvants benefited from their immunomodulatory effects and low cytotoxicity. To point out, approximately all of the adjuvants are first marketed in China as polysaccharides extracted from traditional Chinese medicinal plants or fungi except for Coriolusversicolor polysaccharide and lentinan, which are developed by Japan. Nevertheless, none of the above antitumor adjuvants have been approved by FDA so far.

Drugs for the treatment of blood and cardiovascular diseases account for $22 \%$ of the marketed carbohydrate drugs, which are in the second position as the indications concerned. Fondaparinux, approved by FDA in 2001 , is a fully synthetic analog according to the pentasaccharide domain of heparin and widely used for the treatment of deep vein thrombosis in clinic. It is the first selective antithrombin III (AT III) mediating Xa inhibitor with AT III as its only binding target in plasma 


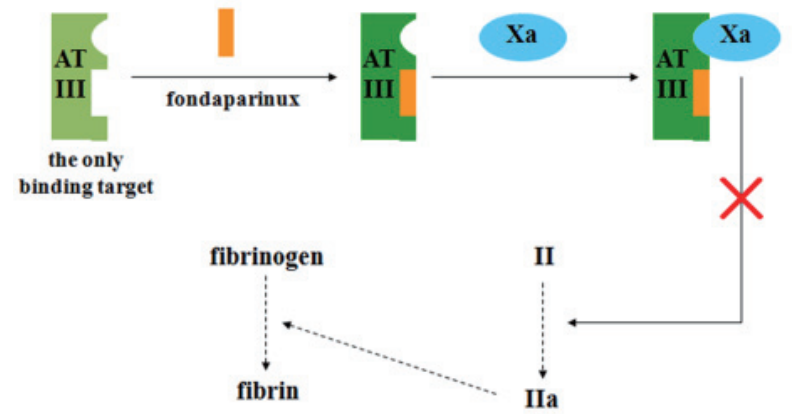

Figure 2. The anticoagulant mechanism of fondaparinux.

compared with unfractionated heparin (UFH) and other low molecular weight heparins (LMWHs) (Figure 2) (15). Consequently, both improved subcutaneous bioavailability and reduced risk of contamination were achieved (16). Voluven, registered by Fresenius Kabi (Bad Homburg, Germany), is a profitable novel hydroxyethyl starch plasma volume expander (17). Reduced coagulation impairment was gained by Voluven with a lower molecular weight $(130 \mathrm{kDa})$ and a lower molar substitution (0.4) compared with Haes (average molecular weight $200 \mathrm{kDa}$, degree of substitution 0.5; Fresenius Kabi) (18). Besides, swine duodenum mucopolysaccharide extracted from the duodenum of healthy swines with a glucosamine content of more than $20.0 \%$, is produced in China as an anti-coronary atherosclerosis pharmaceutical.

Urinary diseases are also a relatively hot field for currently marketed carbohydrate drugs, occupying a portion of $17 \%$. Icodextrin, a cornstarch-derived glucan with a molecular weight of $16 \mathrm{kDa}$, is a biocompatible colloid osmotic agent for peritoneal dialysis for the treatment of chronic renal failure approved by FDA in 2002. Compared with the traditional glucose solution, more stable ultrafiltration rate and lower risk of peritoneal fibrosis were achieved owing to the difficulties in reabsorption and fewer glucose degradation products of icodextrin solution (19-21). Elmiron, developed by Ortho Mcneil Janssen, is the only oral drug approved by FDA for interstitical cystitis. Pentosan polysulfate sodium, the active ingredient of Elmiron, is a semi-synthetic heparin-like derivative (22). It is possibly considered that the glycosaminoglycanlike pentasaccharide of Elmiron can rebuild the highly hydrophilic and protective glycosaminoglycans (GAGs) layer on the bladder urothelium (Figure 3) (23). Hai Kun Shen Xi Capsule, with sulfated fucans as its active ingredient, is another Chinese medicine unapproved by FDA for the treatment of chronic renal failure together with hemodialysis.

Additionally, the physical or biochemical properties of carbohydrates and glycomimetic drugs are also exploited for the treatment of gastro-intestinal diseases, virus infections, and diabetes. Fiberform, registered by Recip $\mathrm{AB}$, is the top choice for constipation recommended by

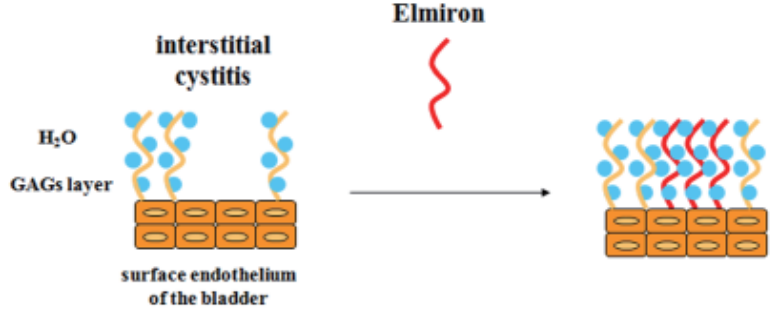

Figure 3. The possible mechanism of Elmiron for the treatment of interstitial cystitis.

the World Gastroenterology Organization. The natural wheat fiber in this pharmaceutical is able to soften the stool by absorbing large amount of water without diarrhea which is the common side effect of other laxatives. The sialic acid mimic, zanamivir (registered by GlaxoSmithKline), is widely used for clinical prevention and treatment of influenza by inhibiting the viral neuraminidase $(24,25)$. Another monosaccharide mimic, miglitol, is designed mimicking the structure of glucose to inhibit the carbohydrate digestion activity of $\alpha$-glucosidase (26-28). Miglitol has been used for the treatment of non-insulin-dependent-diabetes mellitus (NIDDM) since registered by Bayer in 1996.

Noticeably, most of the marketed carbohydrate drugs exhibit favorable pharmacological activities with low cytotoxicity in the treatment of various human diseases based on their physical and biochemical properties. However, the applications of most polysaccharide derived pharmaceuticals such as the polysaccharide antitumor adjuvants, Elmiron, and Hai Kun Shen Xi Capsule are still regional restricted due to their mixed compositions, ambiguous mechanisms, and the difficulties in quality control. Hence, more efforts are needed to put in the structure characterization, pharmacological mechanism investigation, and precise quality control of carbohydrate drugs for their more extensive utilization.

\section{Carbohydrate drugs in development}

Carbohydrate-based therapeutics are extremely potential and a vast number of carbohydrate drugs are in the stage of preclinical or clinical studies. Several carbohydrate drugs in Phase III clinical studies are listed in Table 3.

Cancer is also the hottest field of current carbohydrate drug research and development with several potential therapeutics in development. Synthetic tumor associated carbohydrate antigens are considered to be ideal candidates to elicit antitumor immune responses. Theratope, a conjugation of sialyl Tn oligosaccharide epitope and keyhole limpet hemocyanine (KLH), was developed to be a promising carbohydrate vaccine for metastatic colon cancer and breast cancer. However, the Phase III clinical trial of Theratope failed after ten years of hard work because of its low efficiency (29-32). On the other hand, PI- 88 which is primarily composed of 
Table 3. Examples of carbohydrate drugs in development

\begin{tabular}{|c|c|c|c|}
\hline Drugs & Indications & Institutes & Phase of development \\
\hline Theratope (sialylTn Ag conjugate vaccine) & Metastatic colon cancer, breast cancer & Biomira, Memorial Sloane-Kettering & Phase III discontinued \\
\hline PI-88 & Liver cancer & Progen, Medigen & Phase III \\
\hline Vevesca (Miglustat) & Fabry's disease, Gaucher's disease, HIV & Oxford Glycoscience/Celltech & Phase III \\
\hline Naturlose (D-tagatose) & NIDDM & Spherix & Phase III \\
\hline Betafectin (beta-glucan) & Postoperative infectioin & Alpha-Beta Tech & Phase III \\
\hline Therafectin (amiprilose) & Rheumatoid arthritis & Boston Life Science & Applying \\
\hline
\end{tabular}

highly sulfated oligomannose generated from yeast turns to be the most potential novel antitumor pharmaceutical exhibiting excellent antiangiogenic and anti-metastatic activities (33). PI-88 was initially developed by Progen while the Phase III clinical trial of PI-88 in hepatocellular carcinoma patients has been conducted all around the world by Taiwan Medigen Biotechnology Corporation since 2011 (34-36). Medigen reported that the treatment of patients was nearly completed and desirable results were expected.

Moreover, monosaccharides and their mimics are intensively studied as inhibitors of enzymes associated with carbohydrate metabolism. Vevesca, an orally effective amino sugar, is able to inhibit the glucosyltransferase in the biosynthetic pathway of glycosphingolipid. It has already been approved by FDA for the treatment of mild or moderate type 1 Gaucher's disease in adult, while the anti-HIV trial of Vevesca unfortunately failed in preclinical stage (37-39). Besides, D-tagatose is also proposed to reduce the absorption of carbohydrates by inhibiting the disaccharidases in intestine and interfering with the transportation of glucose (40). It is a promising antidiabetic and obesity controlling pharmaceutical which has achieved statistical significance in a Phase III trial for NIDDM conducted by Spherix (41,42).

Additionally, betafectin, a glucan derived from yeast cell walls, is able to bind with the specific receptors on monocytes and neutrophils, promoting cell proliferation and increasing cell activities (43). Consequently, it is able to significantly prevent postoperative infection by enhancing the immunity of patients.

As described above, numbers of potential carbohydrate drugs in development display promising activities. Challengingly, carbohydrate-based therapeutics including carbohydrate vaccines towards cancers and complicated metabolic diseases are the main tasks of current carbohydrate research and development.

\section{Development prospects of carbohydrate drugs}

There is an increasing interest in the development of carbohydrate candidates for novel drug discovery since most of the currently used carbohydrate drugs display remarkable pharmacological activities and low toxicity. Different approaches can be followed for the discovery and design of carbohydrate drugs $(2,44)$.

\subsection{Discovery of novel carbohydrate drugs}

Naturally occurring polysaccharides and their degradation fragments especially for those generated from traditional Chinese medical herbs and fungi possessing diverse structures and bioactivities are always the most potential library for carbohydrate drug discovery. Several successful carbohydrate pharmaceuticals derived from native glycan structures have already been used in clinic for years, such as LMWHs, alginic acid, hyaluronic acid etc. (45-47). Moreover, a host of natural polysaccharides and oligosaccharides are at the stage of basic research guiding the carbohydrate drug development. For example, polysaccharides from Chinese wolfberry, Agaricusblazei Murill and meretrix are all intensively studied on their anti-tumor, anti-oxidative, and immunomodulatory activities (48-50).

However, the development of most natural polysaccharides encounters difficulties in structure characterization, mechanism unraveling, and quality control, which hamper their universal approval. Besides, the construction of the huge glycan library is also rendered tough by the difficulties to obtain glycans either from natural extraction or from artificial synthesis.

\subsection{Modification of carbohydrates}

There is convincing evidence that appropriate molecular modification of natural glycans could generate novel bioactivities, enhance the original activities, or improve the solubility and pharmacokinetic properties. A lot of efficient methods have been developed for the modification of polysaccharides from various sources.

Sulfation is considered to be one of the most convenient and feasible methods of polysaccharide modification endowing glycans with anti-virus, antitumor, and anti-inflammation activities. A recent investigation demonstrated that curdlan sulfate could effectively inhibit the HBV infection of HepG2 and HepaRG cells and could be developed as a vaccine adjuvant for the treatment or prevention of HBV infection (51,52). SIP-SII, a sulfated Sepiella maindroni ink polysaccharide, demonstrated significant suppressing activity for the metastasis of melanoma by inhibiting tumor adhesion and angiogenesis compared to the unsulfated SIP (53). Besides, selenylation, 
phosphorylation, acetylation, carboxymethylation, and alkylation are all efficient strategies for the modification of polysaccharides such as Atractylodes macrocephala polysaccharide, Chinese angelica polysaccharide, and achyranthes polysaccharide, which endue them varieties of bioactivities $(54,55)$.

\subsection{Carbohydrates mediated drug modification and delivery}

Recently, the specific recognition reactions mediated by saccharide ligands and their specific receptors on the corresponding organs, tissues, and cells are extensively studied for pharmaceutical modification and targeting drug delivery $(2,56,57)$. For example, lactosylated microspheres with high specificity for the asialoglycoprotein lectin expressed on mammalian hepatocytes have been created for liver-specific delivery of drugs and genes $(58,59)$. Mannose modified liposomes selectively targeting their mannose receptors have also been developed as efficient alveolar macrophage selective drug carriers $(60,61)$. Moreover, a novel lectindirected enzyme-activated prodrug therapy (LEAPT) has been developed for regio-selective drug release by carbohydrate-lectin interaction mediated localization of a glycosidase enzyme to the target cells (62).

In addition, natural polysaccharides, as well as their derivatives, are also attractive pharmaceutical and biomedical materials for better solubility, stability and lower toxicity based on their biodegradability, biocompatibility, and non-immunogenic properties $(63,64)$. It has been demonstrated that oligomannose modification of the asparagine residue of C34, a 34mer peptide derived from the C-ectodomain of HIV1 envelope glycoprotein, dramatically improved its solubility and stability as a promising candidate for anti-HIV agents $(65,66)$. Based on the hydrophilic outside surface and the hydrophobic internal cavity, cyclodextrins have been successfully exploited to form inclusion complexes with various hydrophobic drugs to dramatically improve their water solubility (67). Besides, both chitosan and hyaluronic acidare intensively investigated on their unique biomedical properties as novel materials for drug delivery (68).

\subsection{Carbohydrate vaccines}

Both specific polysaccharides located on the surface of pathogenic microorganisms andtumor associated carbohydrate antigens can be specifically recognized by the host immune system and exploited as the basis for the design of carbohydrate vaccines (69-71). Conjugated carbohydrate vaccines have been proved to be effective in the prevention or treatment of a range of human diseases.

By far, several carbohydrate vaccines based on capsular polysaccharides have already been commercialized to preventthe corresponding bacterial or viral infections. For example, Haemophilus influenzae type $\mathrm{B}(\mathrm{HiB})$ conjugate vaccine, a conjugation of purified capsular polysaccharide from $\mathrm{HiB}$ and tetanus toxoid, has been recommended for the prevention of childhood meningitis and recorded in CP and EP. At present, many promising anti-tumor and anti-HIV vaccines are intensively studied with chemically synthesized clusters of oligosaccharide as antigen epitopes. Globo-H, a synthetic hexasaccharide from the cell surface of breast cancer, was covalently linked to KLH with QS21 as an adjuvant and displayed favorable results in the phase I trial $(72,73)$. Carbohydrate vaccines based on oligomannose clusters are also intensively studied for their anti-HIV activities $(74,75)$.

\subsection{Oligosaccharide synthesis}

Chemical or enzymatic synthesis is considered to be the most potential strategy to acquire sufficient amount of homogeneous and structurally well-defined oligosaccharides for carbohydrate drug development due to the limitation of the natural sources. However, unlike polymerase chain reaction (PCR) or solid phase peptide synthesis (SPPS), the synthesis of oligosaccharides is rendered extremely challenging by the structural complexity of carbohydrates without any universal automatic strategy $(76,77)$.

Currently, a great effort is invested in establishing efficient oligosaccharide synthetic approaches and a lot of oligosaccharides have been successfully synthesized such as $\mathrm{Le}^{\mathrm{x}}, \mathrm{Le}^{\mathrm{y}}$, and Globo-H (78-80). However, most of the developed chemical strategies are time consuming and labor intensive with poor region- or stereo-selectivity and low yield in spite of their flexibility (81). On the other hand, enzymatic approaches with high efficiency and selectivity are usually limited by the sources of the enzymes desired. Consequently, chemoenzymatic synthesis which combines the flexibility of the chemical method and the efficiency and selectivity of the enzymatic method is considered to be one of the most efficient approaches for complex oligosaccharide preparation $(82,83)$. A disialyl tetrasaccharide epitope has been regioselectively synthesized based on a chemical manipulation strategy mediated by a bacterial sialyltransferase Pd2,6ST (84). The chemoenzymatic strategy based on bacterial $\beta 1-3$ galactosetransferase and $\alpha 2-3$ sialyltransferase has also been developed for the efficient synthesis of fluorinated T-antigens and their sialylated derivertives (85).

In spite of all the difficulties currently encountered, the successful synthesis of highly pure pentasaccharide, fondaparinux, in multi-kilogram scale has convincingly demonstrated the feasibility and potential of artificial synthesis for industrial production. It is expected that efficient synthetic protocols will be developed, which will open access to various oligosaccharide structures 
for the development of carbohydrate drugs $(76,86)$.

\section{Conclusions}

Despite the structural complexity of carbohydrates, a vast array of carbohydrate drugs have already been included in important pharmacopoeias, marketed, and in development for the treatment of a variety range of human diseases such as cancer, AIDS, diabetes etc. However, more efforts should be put on the study of comprehensive and normalized standards, carbohydrates with defined structures, precise mechanisms and practical quality control for the application and development of carbohydrate drugs.

Additionally, drug discovery and design based on natural occurring glycans, carbohydrate modification, carbohydrate mediated drug modification and delivery, carbohydrate vaccines, and oligosaccharide synthesis are all highly potential approaches for the future development of carbohydrate-based therapeutics. We anticipate that carbohydrate drugs will be one of the most attractive fields of novel drug research and development and play more important roles in the treatment of human diseases in the future.

\section{Acknowledgements}

This work was supported by grants from the National Natural Science Foundation of China (No. 30973678) and the Natural Science Foundation of Shandong Province (ZR2009CZ010).

\section{References}

1. Cai MS, Li ZJ. Carbohydrate Chemistry, Fundamentals, Reactions, Synthesis, Isolations and Structures. Chemical Industry Press, Beijing, China. 2008; pp. 315-368. (in Chinese)

2. Osborn HM, Evans PG, Gemmell N, Osborne SD. Carbohydrate-based therapeutics. J Pharm Pharmacol. 2004; 56:691-702.

3. The Chinese Pharmacopoeia Commission. Chinese Pharmacopoeia 2010. China Medical Science Press, Beijing, China, 2010.

4. Pharmacopoeia Commission of the Chinese Ministry of Health. Drug Standards of the Chinese Ministry of Health. Pharmacopoeia Commission of the Chinese Ministry of Health, Beijing, China, 1998.

5. The United States Pharmacopeial Convention. U. S. Pharmacopoeia/National Formulary (USP 37/NF 32). United States Pharmacopeial Convention, Washington D. C., USA, 2014.

6. The European Pharmacopoeia Commission. European Pharmacopoeia 7.0. The European Directorate for the Quality of Medicines \& HealthCare, Strasbourg, France, 2011.

7. Japanese Pharmacopoeia Committee. Japanese Pharmacopoeia Sixteenth Edition. Pharmaceuticals and Medical Devices Agency, Tokyo, Japan, 2011.

8. Qi JZ, Wang QL, Yu Z, Chen X, Wang FS. Innovative drug R\&D in China. Nat Rev Drug Discov. 2011; 10:333334.

9. Kinch MS, Merkel J. An analysis of FDA-approved drugs for inflammation and autoimmune diseases. Drug Discov Today. 2015; http://dx.doi.org/10.1016/ j.drudis.2015.02.006 (accessed April 26, 2015).

10. Patridge E, Gareiss P, Kinch MS, Hoyer D. An analysis of FDA-approved drugs natural products and their derivatives. Drug Discov Today. 2015; http://dx.doi. org/10.1016/ j.drudis.2015.01.009 (accessed April 26, 2015).

11. Michael SK, Yulia S, Denton H. An analysis of FDAapproved drugs for cardiovascular diseases. Drug Discov Today. 2014; http://dx.doi.org/10.1016/ j.drudis.2014.09.001 (accessed April 26, 2015).

12. China Food and Drug Administration. http://www.sfda. gov.cn/WS01/CL0001/ (accessed April 26, 2015).

13. U. S. Food and Drug Administration. http://www.fda.gov/ (accessed April 26, 2015).

14. Drug future. http://www.drugfuture.com/Index.html (accessed April 26, 2015).

15. Bauer KA, Hawkins DW, Peters PC, Petitou M, Herbert JM, van Boeckel CA, Meuleman DG. Fondaparinux, a synthetic pentasaccharide: the first in a new class of antithrombotic agents - the selective factor Xa inhibitors. Cardiovasc Drug Rev. 2002; 20:37-52.

16. Berqqvist $D$. Review of fondaparinux sodium injection for the preventioin of venous thromboembolism in patients undergoing surgery. Vasc Health Risk Manaq. 2006; 2:365-370.

17. Langeron O, Doelberg M, Ang ET, Bonnet F, Capdevila X, Coriat P. Voluven ${ }^{\circledR}$, a lower substituted novel hydroxyethyl starch (HES 130/0.4), causes fewer effects on coagulation in major orthopedic surgery than HES 200/0.5. Anesth Analg. 2001; 92:855-862.

18. Hitosugi T, Saito T, Suzuki S, Kubota I, Shoda E, Shimizu T, Oi Y. Hydroxyethyl starch: the effect of molecular weight and degree of substitution on intravascular retention in vivo. Anesth Analg. 2007; 105:724-728.

19. Rivera M. Icodextrin as first treatment: reasons to be optimistic. Nefrología. 2009; 29:99-102.

20. Han SH, AhnSV, Yun JY, Tranaeus A, Han DS. Effects of icodextrin on patient survival and technique success in patients undergoing peritoneal dialysis. Nephrol Dial Transplant. 2012; 27:2044-2050.

21. Verco SJ, Peers EM, Brown CB, Rodgers KE, Roda N, diZerega G. Development of a novel glucose polymer solution (icodextrin) for adhesion prevention: pre-clinical studies. Hum Reprod. 2000; 15:1764-1772.

22. Zaslau S, Riggs DR, Jackson BJ, Adkins FC, John CC, Kandzari SJ, McFadden DW. In vitro effects of pentosan polysulfate against malignant breast cells. Am J Surg. 2004; 188:589-592.

23. Metts JF, Davis. Interstitial cystitis: urgency and frequency syndrome. Am Fam Physician. 2001; 64:1199-1206.

24. Feng E, Shin WJ, Zhu X, Li J, Ye D, Wang J, Zheng M, Zuo JP, No KT, Liu X, Zhu W, Tang W, Seong BL, Jiang $\mathrm{H}$, Liu H. Structure-based design and synthesis of C-1and C-4-modified analogs of zanamivir as neuraminidase tnhibitors. J Med Chem. 2013; 56:671-684.

25. Heneghan CJ, OnakpoyaI, Cohen MT, Spencer EA, Jones M, Jefferson T. Zanamivir for influenza in adults and children: systematic review of clinical study reports and summary of regulatory comments. Brit Med J. 2014; 348:2547-2563. 
26. Aoki K, Muraoka T, Ito Y, Togashi Y, Terauchi Y. Comparison of adverse gastrointestinal effects of acarbose and miglitol in healthy men: across over study. Inter Med. 2010; 49:1085-1087.

27. Aoki K, Kamiyama H, Masuda K, Kamiko K, Noguchi Y, Tajima K, Terauchi Y. Effects of miglitol, vildagliptin, or their combination on serum insulin and peptide YY levels and plasma glucose, cholecystokinin, ghrelin, and obestatin levels. Endo J. 2014; 61:249-256.

28. Shimabukuro M, Higa M, Yamakawab K, Kamiko K, Noguchi Y, Tajima K, Terauchi Y. Miglitol, $\alpha$-glycosidase inhibitor, reduces visceral fat accumulation and cardiovascular risk factors in subjects with the metabolic syndrome: A randomized comparable study. Int J Cardiol. 2013; 2108-2113.

29. Adis International Ltd. Cancer vaccine THERATOPEBiomira. Drugs R D. 2003; 4:236-240.

30. Gilewski TA, Ragupathi G, Dickler M, Powell S, Bhuta S, Panageas K, Koganty RR, Chin-Eng J, Hudis C, Norton L, Houghton AN, Livingston PO. Immunization of high-risk breast cancer patients with clustered sTn- KLH conjugate plus the immunologic adjuvant QS-21. Clin Cancer Res. 2007; 13:2977-2985.

31. Holmberg LA, Sandmaier BM. Vaccination with Theratope (STn-KLH) as treatment for breast cancer. Expert Rev Vaccines. 2004; 3:655-663.

32. Holmberg LA, Sandmaier BM. Theratope vaccine (STnKLH). Expert Opin Biol Ther. 2001; 1:881-891.

33. Scott EN, Thomas AL. PI-88. Heparanase inhibitor, antiangiogenic agent, oncolytic. Drug Future. 2008; 33: 21.

34. Liu CJ, Chang J, Lee PH, et al. Adjuvant heparanase inhibitor PI-88 therapy for hepatocellular carcinoma recurrence. World J Gastroenterol. 2014; 20:11384-11393.

35. Lewis KD, Robinson WA, Millward MJ, Powell A, Price TJ, Thomson DB, Walpole ET, Haydon AM, Creese BR, Roberts KL, Zalcberg JR, Gonzalez R. A phase II study of the heparanase inhibitor PI- 88 in patients with advanced melanoma. Invest New Drugs. 2008; 26:89-94.

36. Khasraw M, Pavlakis N, McCowatt S, Underhill C, Begbie S, de Souza P, Boyce A, Parnis F, Lim V, Harvie R, Marx G. Multicentrephase I/II study of PI-88, a heparanase inhibitor in combination with docetaxel in patients with metastatic castrate-resistant prostate cancer. Ann Oncol. 2010; 21:1302-1307.

37. Cox TM, Aerts JM, Andria G, et al. The role of the iminosugar N-butyldeoxynojirimycin (Miglustat) in the management of type I (nonneuronopathic) Gaucher' disease: a position statement. JInherit Metab Dis. 2003; 26:513-526.

38. Kuter DJ, Mehta A, Hollak CE, Giraldo P, Hughes D, Belmatoug N, Brand M, Muller A, Schaaf B, Giorgino R, Zimran A. Miglustat therapy in type 1 Gaucher disease: clinical and safety outcomes in a multicenter retrospective cohort study. Blood Cells Mol Dis. 2013; 51:116-124.

39. Venier RE, Igdoura SA. Miglustat as a therapeutic agent: prospects and caveats. J Med Genet. 2012; 49:591-597.

40. Lu Y, Levin GV, Donner TW. Tagatose, a new antidiabetic and obesity control drug. Diabetes Obes Metab. 2008; 10:109-134.

41. Espinosa I, Fogelfeld L. Tagatose: from a sweetener to a new diabetic medication? Expert Opin Investig Drugs. 2010; 19:285-294.

42. Jennifer B. Spherixsoars on D-tagatoseresults, starts partner hunt. Bio World Today. 2010; 21: 1.
43. Dellinger EP, BabineauTJ, Bleicher P, Kaiser AB, Seibert GB, Postier RG, Vogel SB, Norman J, Kaufman D, Galandiuk S, Condon RE. Effect of PGG-glucan on the rate of serious postoperative infection or death observed after high-risk gastrointestinal operations. Betafectin Gastrointestinal Study Group. Arch Surg. 1999; 134:977983.

44. Cipolla L, Araújo AC, Bini D, Gabrielli L, Russo L, Shaikh N. Discovery and design of carbohydrate-based therapeutics. Expert Opin Drug Discov. 2010; 5:721-737.

45. Holzheimer RG. Low-molecular-weight heparin (LMWH) in the treatment of thrombosis. Eur J Med Res. 2004; 9:225-239.

46. Nicolau JC, Cohen M, Montalescot. Differences among low-molecular-weight heparins: evidence in patients with acute coronary syndromes. J Cardiovasc Pharmacol. 2009; 53:440-445.

47. Vogel R, Crockett RS, Oden N, Laliberte TW, Molina L. Demonstration of efficacy in the treatment of dry eye disease with $0.18 \%$ sodium hyaluronate ophthalmic solution (vismed, rejena). Am J Ophthalmol. 2010; 149:594-601.

48. Cui L, Sun Y, Xu H, Cong H, Liu J. A polysaccharide isolated from Agaricusblazei Murill (ABP-AW1) as a potential Th1 immunity-stimulating adjuvant. Oncol Lett. 2013; 6:1039-1044.

49. Li X, Zhao X, Wang H, Han J, Liu L. A polysaccharide from the fruiting bodies of Agaricusblazei Murill induces caspase-dependent apoptosis in human leukemia HL-60 cells. Tumour Biol. 2014; 35:8963-8968.

50. Liu SX, Qi ZH, Zhang JJ, He CB, Gao XG, Li HJ. Lipopolysaccharide and $\beta$-1,3-glucan binding protein in the hard clam (Meretrix meretrix): Molecular characterization and expression analysis. Genet Mol Res. 2014; 13:4956-4966.

51. Li PL, Zhang XK, Cheng YN, Li J, Xiao Y, Zhang Q, Zong A, Zhong C, Wang F. Preparation and in vitro immunomodulatory effect of curdlan sulfate. Carbohyd Polym. 2014; 102:852-861.

52. Li PL, Tan HN, Xu DQ, Yin F, Cheng Y, Zhang X, Liu Y, Wang F. Effect and mechanisms of curdlan sulfate on inhibiting $\mathrm{HBV}$ infection and acting as an $\mathrm{HB}$ vaccine adjuvant. Carbohyd Polym. 2014; 110:446-455.

53. Zong AZ, Zhao T, Zhang Y, Song X, Shi Y, Cao H, Liu C, Cheng Y, Qu X, Cao J, Wang F. Anti-metastatic and antiangiogenic activities of sulfated polysaccharide of Sepiella maindroni ink. Carbohyd Polym. 2013; 91:403-409.

54. Qin T, Chen J, Wang DY, Hu Y, Zhang J, Wang M, Qiu S, Gao Z, liu R, Yu Y, Huang Y, Wang Q, Wang Q. Selenylation modification can enhance immune-enhancing activity of Chinese angelica polysaccharide. Carbohyd Polym. 2013; 95:183-187.

55. Liu J,Chen X, Yue CJ, et al. Effect of selenylation modification on immune-enhancing activity of Atractylodes macrocephala polysaccharide. Int J Biol Macromol. 2015; 72:1435-1440.

56. Wirth M, Fuchs A, Wolf M, Ertl B, Gabor F. Lectin mediated drug targeting: Preparation, binding characteristics and antiprolifer ative activity of wheat germ agglutinin conjugated doxorubicin on Caco-2 cells. Pharm Res. 1998; 15:1031-1037.

57. Yu WY, Zhang N, Li CJ. Saccharide modified pharmaceutical nanocarriers for targeted drug and gene delivery. Curr Pharm Des. 2009; 15:3826-3836.

58. Vyas SP, Singh A, Sihorkar V. Ligand-receptor mediated 
drug delivery: An emerging paradigm in cellular drug targeting. Crit Rev Ther Drug Carrier Syst. 2001; 18:1-76.

59. Davis BG, Robinson MA. Drug delivery systems based on sugar-macromolecule conjugates. Curr Opin Drug Discov Dev. 2002; 5:279-288.

60. Lu Y, Kawakami S, Yamashita F, Hashida M. Development of an antigen-presenting cell-targeted DNA vaccine against melanoma by mannosylated liposomes. Biomaterials. 2007; 28:3255-3262.

61. Tiwari S, Chaturvedi AP, Tripathi YB, Mishra B. Macrophage-specific targeting of isoniazid through mannosylated gelatin microspheres. AAPS Parm Sci Tech. 2011; 12:900-908.

62. Davis, B. Preparation of enzyme conjugated to carbohydrate moiety which binds to lectin-directed prodrug delivery system. 2002; PCT Int. Appl. Patent No.WO02/080980.

63. Baldwin AD, Kiick KL. Polysaccharide-modified synthetic polymeric biomaterials. Biopolymers. 2010; 94:128-140

64. Lemarchand C, Gref R, Couvreur P. Polysaccharidedecorated nanoparticles. Eur J Pharm Biopharm. 2004; 58:327-341.

65. Huang W, Groothuys S, Heredia A, Kuijpers BH, Rutjes FP, van Delft FL, Wang LX. Enzymatic glycosylation of triazole-linked GlcNAc/Glc-peptides: Synthesis, stability and anti HIV activity of triazole-linked HIV-1 gp41 glycopeptide C34 analogues. Chembiochem. 2009; 10:1234-1242.

66. Wang LX, Song HJ, Liu SW, Lu H, Jiang S, Ni J, Li H. Chemoenzymatic synthesis of HIV-1 gp41 glycopeptides: Effects of glycosylation on the anti-HIV activity and a-helix bundle-forming ability of peptide C34. Chembiochem. 2005; 6:1068-1074.

67. Chen J, Lu WL, Gu W, Lu SS, Chen ZP, Cai BC, Yang $\mathrm{XX}$. Drug-in-cyclodextrin- in-liposomes: A promising delivery system for hydrophobic drugs. Expert Opin Drug Deliv. 2014; 11:565-577.

68. Morris G, Kök S, Harding S, Adams G. Polysaccharide drug delivery systems based on pectin and chitosan. Biotechnol Genet Eng Rev. 2010; 27:257-284.

69. Johannes FG, Vliegenthart. Carbohydrate based vaccines. FEBS Letters. 2006; 580:2945-2950.

70. Huang YL, Wu CY. Carbohydrate-based vaccines: Challenges and opportunities. Expert Rev Vaccines. 2010; 9:1257-1274.

71. Oppenheimer SB, Alvarez M, Nnoli J. Carbohydratebased experimental therapeutics for cancer, HIV/AIDS and other diseases. Acta Histochem, 2008; 110:6-13.

72. Gilewski T, Ragupathi G, Bhuta S, Williams LJ, Musselli C, Zhang XF, Bornmann WG, Spassova M, Bencsath KP, Panageas KS, Chin J, Hudis CA, Norton L, Houghton AN, Livingston PO, Danishefsky SJ. Immunization of metastatic breast cancer patients with a fully synthetic globo H conjugate: A phase I trial. Proc Natl Acad Sci U S A. 2001; 98:3270-3275.

73. Jeon I, Iyer K, DanishefskySJ. A practical total synthesis of globo-H for use in anticancer vaccine. J Org Chem. 2009; 74:8452-8455.

74. Wang LX, Ni JH, Singh S, Li H. Binding of highmannose-type oligosaccharides and synthetic oligomannose clusters to human antibody $2 \mathrm{G} 12$ : Implications for HIV-1 vaccine design. Chem Biol. 2004; 11:127-134.

75. Zhang Y, Chen CC, Tan HN, Wang FS, Cao HZ. Synthesis of unsymmetrical 3,6-branched Man5 oligosaccharide: A comparison between one-pot sequential glycosylation and stepwise synthesis. Carbohydr Res. 2015; 401:109-114.

76. Seeberger PH, Werz DB. Automated synthesis of oligosaccharides as a basis for drug discovery. Nat Rev Drug Discov. 2005; 4:751-763.

77. Galan MC, Alifonso DB, Watt GM. Carbohydrate chemistry in drug discovery. Org Biomol Chem. 2011; 9:3598-3610.

78. Otsubo N, Ishida H, Kiso M, Hasegawa A. Sythesis of sialyl Le ${ }^{\mathrm{X}}$ ganglioside analogues modified at C-6 of the galactose residue to elucidate the mechanism of selection recognition. Carbohydr Res. 1998; 306:517-530.

79. Spassova MK, BornmannWG, Ragupathi G, Sukenick G, Livingston PO, Danishefsky SJ. Synthesis of selected Le ${ }^{\mathrm{Y}}$ and KH-1 analogues: A medicinal chemistry approach to vaccine optimization. J Org Chem. 2005; 70:3383-3395.

80. Jeon I, Iyer K, Danishefsky SJ. A practical total synthesis of globo-H for use in anticancer vaccines. J Org Chem. 2009; 74:8452-8455.

81. Zhu X, Schmidt RR. New principles for glycoside-bond formation. Angew Chem Int Ed Engl. 2009; 48:19001934.

82. Filice M, Marciello M. Enzymatic synthesis of oligosaccharides: A powerful tool for a sweet challenge. Curr Org Chem. 2013; 17:701-718.

83. Weijers CA, Franssen M C, Visser GM. Glycosyltransferasecatalyzed synthesis of bioactive oligosaccharides. Biotechnol Adv. 2008; 26:436-456.

84. Meng X, Yao W, Cheng J, Jin L, Yu H, Chen X, Wang F, Cao H. Regioselective chemoenzymatic synthesis of ganglioside disialyl tetrasaccharide epitopes. J Am Chem Soc. 2014; 136:5205-5208.

85. Yan J, Chen X, Wang FS, Cao HZ. Chemoenzymatic synthesis of mono- and di-fluorinated ThomsenFriedenreich $(\mathrm{T})$ antigens and their sialylated derivatives. Org Biomol Chem. 2013; 11:842-848.

86. Tiwari VK, Mishra RC, Sharma A, Tripathi RP. Carbohydrate based potential chemotherapeutic agents: Recent developments and their scope in future drug discovery. Mini Rev Med Chem. 2012; 12:1497-1519.

(Received April 12, 2015; Accepted April 27, 2015) 